\title{
Obstacle Avoidance Using Haptics and a Laser Rangefinder
}

\author{
Daniel Innala Ahlmark*, Håkan Fredriksson*, Kalevi Hyyppä*
}

\begin{abstract}
In its current form, the white cane has been used by visually impaired people for almost a century. It is one of the most basic yet useful navigation aids, mainly because of its simplicity and intuitive usage. For people who have a motion impairment in addition to a visual one, requiring a wheelchair or a walker, the white cane is impractical, leading to human assistance being a necessity. This paper presents the prototype of a virtual white cane using a laser rangefinder to scan the environment and a haptic interface to present this information to the user. Using the virtual white cane, the user is able to "poke" at obstacles several meters ahead and without physical contact with the obstacle. By using a haptic interface, the interaction is very similar to how a regular white cane is used. This paper also presents the results from an initial field trial conducted with six people with a visual impairment.
\end{abstract}

\section{INTRODUCTION}

During the last few decades, people with a visual impairment have benefited greatly from the technological development. Assistive technologies have made it possible for children with a visual impairment to do schoolwork along with their sighted classmates, and later pick a career from a list that-largely due to assistive technologies-is expanding. Technological innovations specifically designed for people with a visual impairment also aid in daily tasks, boosting confidence and independence.

While recent development has made it possible for a person with a visual impairment to navigate the web with ease, navigating the physical world is still a major challenge. The white cane is still the obvious aid to use. It is easy to operate and trust because it behaves like an extended arm. The cane also provides auditory information that helps with identifying the touched material as well as acoustic echolocation. For someone who, in addition to a visual impairment, is in need of a wheelchair or a walker, the cane is impractical to use and therefore navigating independently of another person might be an impossible task. The system presented in this paper, henceforth referred to as 'the virtual white cane', is an attempt to address this problem using haptic technology and a laser rangefinder. This system makes it possible to detect obstacles without physically hitting them, and the length of the virtual cane can be varied based on user preference and situational needs. Figure 1 shows the system in use.

* \{daniel.innala, hakan.fredriksson, kalevi.hyyppa\}@ltu.se Dept. of Computer Science, Electrical and Space Engineering Luleå University of Technology, Luleå, Sweden

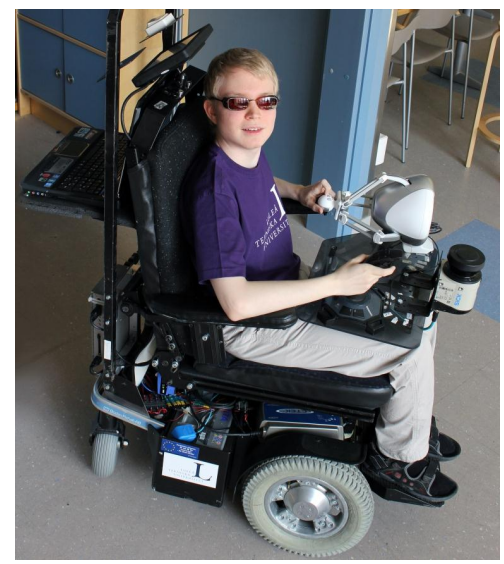

Fig. 1. The virtual white cane. This figure depicts the system currently set up on the MICA wheelchair.

Haptic technology (the technology of the sense of touch) opens up new possibilities of human-machine interaction. Haptics can be used to enhance the experience of a virtual world when coupled with other modalities such as sight and sound [1], as well as for many standalone applications such as surgical simulations [2]. Haptic technology also paves way for innovative applications in the field of assistive technology. People with a visual impairment use the sense of touch extensively; reading braille and navigating with a white cane are two diverse scenarios where feedback through touch is the common element. Using a haptic interface, a person with a visual impairment can experience three-dimensional models without the need to have a physical model built. For the virtual white cane, a haptic interface was a natural choice as the interaction resembles the way a regular white cane is used. This should result in a system that is intuitive to use for someone who has previous experience using a traditional white cane.

The next section discusses previous work concerning haptics and obstacle avoidance systems for people with a visually impairment. Section 3 is devoted to the hardware and software architecture of the system. Section 4 presents results from an initial field trial, and section 5 concludes the paper and gives some pointers to future work.

\section{RELATED WORK}

The idea of presenting visual information to people with a visual impairment through a haptic interface is an appealing one. This idea has been applied to a number of different scenarios during recent years. Fritz et al. [3] 
used haptic interaction to present scientific data, while Moustakas et al. [4] applied the idea to maps.

Models that are changing in time pose additional challenges. The problem of rendering dynamic objects haptically was investigated by e.g. Diego Ruspini and Oussama Khatib [5], who built a system capable of rendering dynamic models, albeit with many restrictions. When presenting dynamic information (such as in our case a model of the immediate environment) through a haptic interface, care must be taken to minimize a phenomenon referred to as haptic fall-through, where it is sometimes possible to end up behind (fall through) a solid surface (see section III-C for more details). Minimizing this is of critical importance in applications where the user does not see the screen, as it would be difficult to realize that the haptic probe is behind a surface. Gunnar Jansson at Uppsala University in Sweden has studied basic issues concerning visually impaired peoples' use of haptic displays [6]. He notes that being able to look at a visual display while operating the haptic device increases the performance with said device significantly. The difficulty lies in the fact that there is only one point of contact between the virtual model and the user.

When it comes to sensing the environment numerous possibilities exist. Ultrasound has been used in devices such as the UltraCane [7], and Yan and Manduchi [8] used a laser rangefinder in a triangulation approach by surface tracking. Depth-measuring (3D) cameras are appealing, but presently have a narrow field of view, relatively low accuracy, and a limited range compared to laser rangefinders. These cameras undergo constant improvements and will likely be a viable alternative in a few years. Indeed, consumer-grade devices such as the Microsoft Kinect has been employed as range-sensors for mobile robots (see e.g. [9]). The Kinect is relatively cheap, but suffers from the same problems as other $3 \mathrm{D}$ cameras at present [10].

Spatial information as used in navigation and obstacle avoidance systems can be conveyed in a number of ways. This is a primary issue when designing a system specifically for the visually impaired, perhaps evidencing the fact that not many systems are widely adopted despite many having been developed. Speech has often been used, and while it is a viable option in many cases, it is difficult to present spatial information accurately through speech [11]. Additionally, interpreting speech is time-consuming and requires a lot of mental effort [12]. Using non-speech auditory signals can speed up the process, but care must be taken in how this audio is presented to the user, as headphones make it more difficult to perceive useful sounds from the environment [13].

\section{THE VIRTUAL WHITE CANE}

Published studies on the subject of obstacle avoidance utilizing force feedback [14], [15] indicate that adding force feedback to steering controls leads to fewer collisions and a better user experience. The virtual white

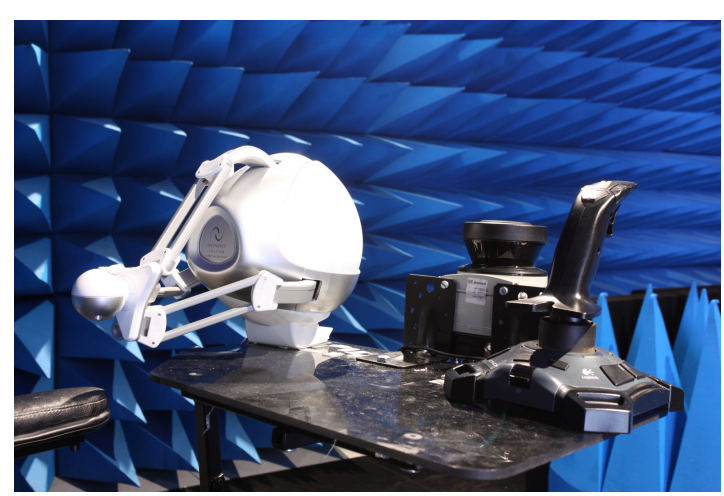

Fig. 2. The Novint Falcon, joystick and SICK LMS111.

cane presented in this paper provides haptic feedback decoupled from the steering process, so that a person with a visual impairment can "poke" at the environment like when using a white cane. Some important considerations when designing such a system are:

- Reliability. A system behaving unexpectedly immediately decreases the trust of said system and might even cause an accident. To become adopted, the benefit and reliability must outweigh the risk and effort associated with using the system. If some problem should arise, the user should immediately be alerted; an error message displayed on a computer monitor is not sufficient.

- Ease of use. The system should be intuitive to use. This factor is especially valuable in an obstacle avoidance system because human beings know how to avoid obstacles intuitively. Minimized training and better adoption of the technology should follow from an intuitive design.

- The system should respond as quickly as possible to changes in the environment. This feature has been the focus for our current prototype. Providing immediate haptic feedback through a haptic interface turned out to be a challenge (see section III-C).

\section{A. Hardware}

The virtual white cane consists of a haptic display (Novint Falcon [16]), a laser rangefinder (SICK LMS111 [17]), and a laptop (MSI GT663R [18] with an Intel Core i7-740QM running at $1.73 \mathrm{GHz}, 8 \mathrm{~GB}$ RAM and an NVIDIA GeForce GTX 460M graphics card). These components, depicted in figure 2, are currently mounted on the electric wheelchair MICA (Mobile Internet Connected Assistant), which has been used for numerous research projects at Luleå University of Technology over the years [19]-[21]. MICA is steered using a joystick in one hand, and the Falcon is used to feel the environment with the other.

The laser rangefinder is mounted so that it scans a horizontal plane of 270 degrees in front of the wheelchair. The distance information is transmitted to the laptop over an ethernet connection at $50 \mathrm{~Hz}$ and contains 541 
angle-distance pairs $(\theta, r)$, yielding an angular resolution of half a degree. The LMS111 can measure distances up to 20 meters with an error within three centimeters. This information is used by the software to build a threedimensional representation of the environment. This representation assumes that for each angle $\theta$, the range $r$ will be the same regardless of height. This assumption works fairly well in a corridor environment where most potential obstacles that could be missed are stacked against the walls. This representation is then displayed graphically as well as transmitted to the haptic device, enabling the user to touch the environment continuously.

\section{B. Software Architecture}

The software is built on the open-source H3DAPI platform which is developed by SenseGraphics AB [22]. $\mathrm{H} 3 \mathrm{D}$ is a scenegraph-API based on the X3D 3D-graphics standard, enabling rapid construction of haptics-enabled $3 \mathrm{D}$ scenes. At the core of such an API is the scenegraph: a tree-like data structure where each node can be defining anything from global properties and scene lighting to properties of geometric objects as well as the objects themselves. To render a scene described by a scenegraph, the program traverses this graph, rendering each node as it is encountered. This concept makes it easy to perform a common action on multiple nodes by letting them be child nodes of a node containing the action. For example, in order to move a group of geometric objects a certain distance, it is sufficient to let the geometric nodes be children of a transform node defining the translation.

H3DAPI provides the possibility of extension through custom-written program modules (which are scenegraph nodes). These nodes can either be defined in scripts (using the Python language), or compiled into dynamically linked libraries from $\mathrm{C}++$ source code. Our current implementation uses a customized node defined in a Python script that repeatedly gets new data from the laser rangefinder and renders it.

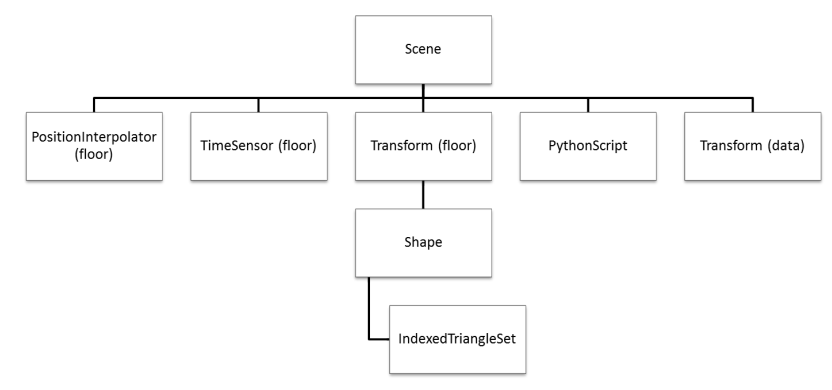

Fig. 3. The X3D scenegraph. This diagram shows the nodes of the scene and the relationship among them. The transform (data) node is passed as a reference to the Python script (described below). Note that nodes containing configuration information or lighting settings are omitted.

1) Scenegraph View: The X3D scenegraph, depicted in figure 3, contains configuration information comprised of haptic rendering settings (see section III-C) as well as properties of static objects. Since the bottom of the Novint Falcon's workspace is not flat, a "floor" is drawn at a height where maximum horizontal motion of the Falcon's handle is possible without any bumps. This makes using the system more intuitive since this artificial floor behaves like the real floor, and the user can focus on finding obstacles without getting distracted by the shape of the haptic workspace. At program start up, this floor is drawn at a low (outside the haptic workspace) height, and is then moved slowly upwards to the designated floor coordinate in a couple of seconds. This movement is done to make sure the haptic proxy (the rendered sphere representing the position of the haptic device) does not end up underneath the floor when the program starts.

The scenegraph also contains a Python script node. This script handles all dynamics of the program by overriding the node's traverseSG method. This method executes once every scenegraph loop, making it possible to use it for obtaining, filtering and rendering new range data.

2) Python Script: The Python script fetches data from the laser rangefinder continually, then builds and renders the model of this data graphically and haptically. It renders the data by creating an indexed triangle set node and attaching it to the transform (data) node it gets from the scenegraph.

The model can be thought of as a set of tall, connected rectangles where each rectangle is positioned and angled based on two adjacent laser measurements. Below is a simplified version of the algorithm buildModel, which outputs a set of vertices representing the model. From this list of points, the wall segments are built as shown in figure 4. For rendering purposes, each tall rectangle is divided into two triangles. The coordinate system is defined as follows: Sitting in the wheelchair, the positive $\mathrm{x}$-axis is to the right, $\mathrm{y}$-axis is up and the z-axis points backwards.

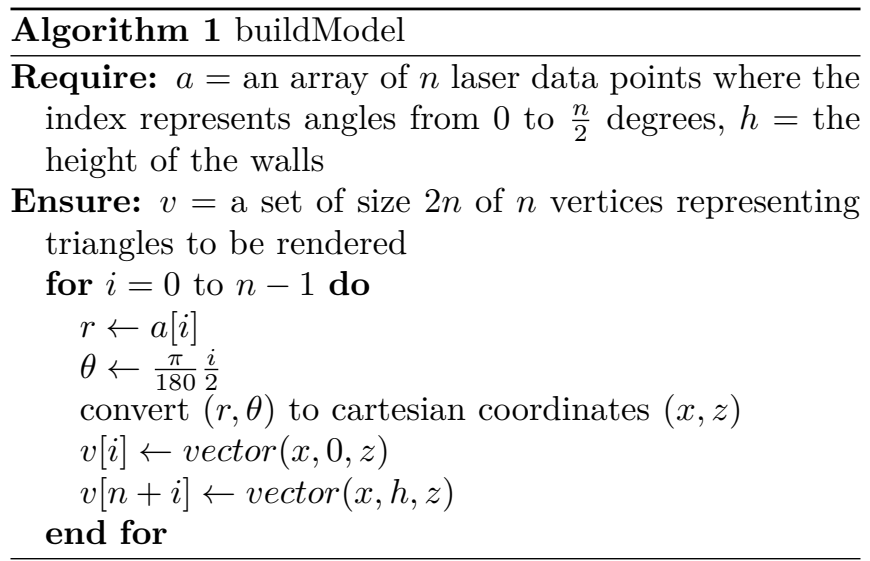

In our current implementation, laser data is passed through three filters before the model is built. These filters - a spatial low-pass filter, a spatial median filter and a time-domain median filter-serve two purposes: Firstly, the laser data is subject to some noise which is 


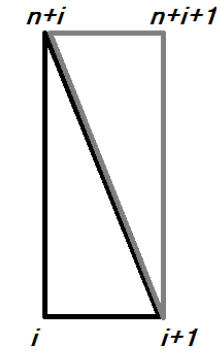

Fig. 4. The $i$ th wall segment, internally composed of two triangles.

noticeable visually and haptically. Secondly, the filters are used to prevent too sudden changes to the model in order to minimize haptic fall-through (see the next section for an explanation of this).

\section{Dynamic Haptic Feedback}

The biggest challenge so far has been to provide satisfactory continual haptic feedback. The haptic display of dynamically changing nontrivial models is an area of haptic rendering that could see much improvement. The most prominent issue is the fall-through phenomenon where the haptic proxy goes through a moving object. When an object is deforming or moving rapidly, time instances occur where the haptic probe is moved to a position where there is no triangle to intercept it at the current instant in time, thus no force is sent to the haptic device. This issue is critical in an obstacle avoidance system such as the virtual white cane where the user does not see the screen, thus having a harder time detecting fall-through.

To minimize the occurrence of fall-through, three actions have been taken:

- Haptic renderer has been chosen with this issue in mind. The renderer chosen for the virtual white cane was created by Diego Ruspini [23]. This renderer treats the proxy as a sphere rather than a single point (usually referred to as a god-object), which made a big difference when it came to fall-through. The proxy radius had a large influence on this problem; a large proxy can cope better with larger changes in the model since it is less likely that a change is bigger than the proxy radius. On the other hand, the larger the proxy is, the less haptic resolution is possible.

- Any large coordinate changes are linearly interpolated over time. This means that sudden changes are smoothed out, preventing a change that would be bigger than the proxy. As a trade-off, any rapid and large changes in the model will be unnecessarily delayed.

- Three different filters (spatial low-pass and median, time-domain median) are applied to the data to remove spuriouses and reduce fast changes. These filters delays all changes in the model slightly, and has some impact on the application's frame rate.

Having these restrictions in place avoids most fallthrough problems, but does so at the cost of haptic resolution and a slow-reacting model, which has been acceptable in the early tests.

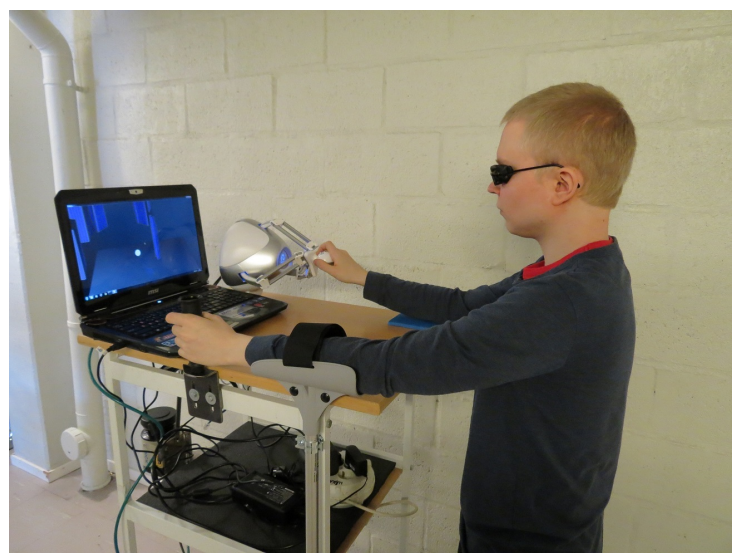

Fig. 5. The virtual white cane as mounted on a movable table. The left hand is used to steer the table while the right hand probes the environment through the haptic interface.

\section{FIELD TRIAL}

In order to assess the feasibility of haptics as a means of presenting information about nearby obstacles to people with a visual impairment, a field trial with six participants (ages 52-83) was conducted. All participants were blind (one since birth) and were white cane users. Since none of the participants were used to a wheelchair, the system was mounted on a table on wheels (see figure 5). A crutch handle with support for the arm was attached to the left side of the table (from the user's perspective) so that it could be steered with the left hand and arm, while the right hand used the haptic interface.

The trial took place in a corridor environment at the Luleå University of Technology campus. The trial consisted of an acquaintance phase of a few minutes where the participants learnt how to use the system, and a second phase where they were to traverse a couple of corridors, trying to stay clear of the walls and avoiding doors and other obstacles along the way. The second phases were video-recorded, and the participants were interviewed afterwards.

All users grasped the idea of how to use the system very quickly. When interviewed, they stated that they thought their previous white cane experience helped them use this system. This supports the notion that the virtual white cane is intuitive to use and easy to understand for someone who is familiar with the white cane. While the participants understood how to use the system, they had difficulties accurately determining the distances and angles to obstacles they touched. This made it tricky to perform maneuvers that require high precision such as passing through doorways. It is worth 
noting that the participants quickly adopted their own technique of using the system. Most notably, a pattern emerged where a user would trace back and forth along one wall, then sweep (at a close distance) to the other wall, and repeated this procedure starting from this wall.

None of the users expressed discomfort or insecurity, but comments were made regarding the clumsiness of the prototype and that it required both physical and mental effort to use. An upcoming article (see [24]; title may change) will present a more detailed report on the field trial.

\section{CONCLUSIONS}

Figure 6 shows a screenshot of the application in use. The field trial demonstrated the feasibility of haptic interaction for obstacle avoidance, but many areas of improvement were also identified. The difficulty in determining the precise location of obstacles could be due to the fact that none of the users had practiced this earlier. Since a small movement of the haptic grip translates to a larger motion in the physical world, a scale factor between the real world and the model has to be learned. This is further complicated by the placement of the laser rangefinder and haptic device relative to the user. As the model is viewed through the perspective of the laser rangefinder, and perceived through a directionless grip held with the right hand, a translation has to be learned in addition to the scale factor in order to properly match the model with the real world. A practice phase specifically made for learning this correspondence might be in order, however, the point of the performed field trial was to provide as little training as possible.

The way the model is built and the restrictions placed on it in order to minimize haptic fall-through have several drawbacks. Since the obstacle model is built as a coherent, deformable surface, a moving object such as a person walking slowly from side to side in front of the laser rangefinder will cause large, rapid changes in the model. As the person moves, rectangles representing obstacles farther back are rapidly shifted forward to represent the person, and vice versa. This means that even some slow motions are unnecessarily delayed in the model as its rate of deformation is restricted. Since the haptic proxy is a large sphere, the spatial resolution that can be perceived is also limited.

\section{A. Future Work}

The virtual white cane is still in its early development stage. Below are some pointers to future work:

- Data acquisition. Some other sensor(s) should be used in order to gather real three-dimensional measurements. 3D time-of-flight cameras look promising but are currently too limited in field of view and signal to noise ratio for this application.

- Haptic feedback. The most prominent problem with the current system regarding haptics is haptic

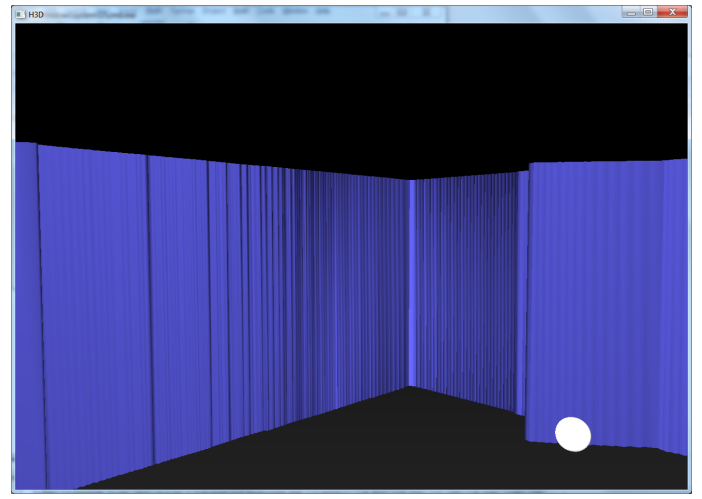

Fig. 6. The virtual white cane in use. This is a screenshot of the application depicting a corner of an office, with a door being slightly open. The user's "cane tip", represented by the white sphere, is exploring this door.

fall-through. The current approach of interpolating changes avoids most fall-through problems but severely degrades the user experience in several ways. One solution is to use a two-dimensional tactile display instead of a haptic interface such as the Falcon. Such displays have been explored in many forms over the years [25]-[27]. One big advantage of such displays is that multiple fingers can be used to feel the model at once. Also, fall-through would not be an issue. On the flip side, the inability of such displays to display three-dimensional information and their current state of development makes haptic interfaces such as the Falcon a better choice under present circumstances.

- Data model and performance. At present the model is built as a single deformable object. Performance is likely suffering because of this. Different strategies to represent the data should be investigated. This issue becomes critical once three-dimensional information is available due in part to the greater amount of information itself but also because of the filtering that needs to be performed.

- Ease of use. A user study focusing on model settings (scale and translation primarily) may lead to some average settings that work best for most users, thus reducing training times further for a large subset of users.

- Other interfaces. It might be beneficial to add additional interaction means (e.g. auditory cues) to the system. These could be used to alert the user that they are about to collide with an obstacle. Such a feature becomes more useful when a full threedimensional model of the surroundings is available. Additionally, auditory feedback has been shown to have an effect on haptic perception [28].

\section{ACKNOWLEDGMENT}

This work was supported by Centrum för medicinsk teknik och fysik (CMTF) at Umeå University and Luleå University of Technology-both in Sweden-and by the 
European Union Objective 2 North Sweden structural fund.

\section{REFERENCES}

[1] A. Lécuyer, P. Mobuchon, C. Mégard, J. Perret, C. Andriot, and J. pierre Colinot, "Homere: a multimodal system for visually impaired people to explore virtual environments," in Proc. IEEE VR, 2003, pp. 251-258.

[2] M. Eriksson, M. Dixon, and J. Wikander, "A haptic VR milling surgery simulator-using high-resolution CT-data," Stud. Health, Technol., Inform., vol. 119, pp. 138-143, 2006.

[3] J. P. Fritz, T. P. Way, and K. E. Barner, "Haptic representation of scientific data for visually impaired or blind persons," in Technology and Persons With Disabilities Conf., 1996.

[4] K. Moustakas, G. Nikolakis, K. Kostopoulos, D. Tzovaras, and M. Strintzis, "Haptic rendering of visual data for the visually impaired," Multimedia, IEEE, vol. 14, no. 1, pp. 62-72, JanMar 2007.

[5] D. Ruspini and O. Khatib, "Dynamic models for haptic rendering systems," accessed 2013-06-18. [Online]. Available: http://citeseerx.ist.psu.edu/viewdoc/download?doi=10. 1.1.127.5804\&rep $=$ rep1\&type $=$ pdf

[6] G. Jansson, "Basic issues concerning visually impaired people's use of haptic displays," in The 3rd International Conf. Disability, Virtual Reality and Assoc. Technol., Alghero, Sardinia, Italy, Sep. 2000, pp. 33-38.

[7] Sound Foresight Technology Ltd, "Ultracane - putting the world at your fingertips," accessed 2013-06-18. [Online]. Available: http://www.ultracane.com/

[8] D. Yuan and R. Manduchi, "Dynamic environment exploration using a virtual white cane," in Proc. 2005 IEEE Computer Society Conf. Computer Vision and Pattern Recognition (CVPR'05). Washington, DC, USA: IEEE Computer Society, 2005, pp. 243-249.

[9] D. Correa, D. Sciotti, M. Prado, D. Sales, D. Wolf, and F. Osorio, "Mobile robots navigation in indoor environments using kinect sensor," in 2012 Second Brazilian Conf. Critical Embedded Systems (CBSEC), May 2012, pp. 36-41.

[10] K. Khoshelham and S. O. Elberink, "Accuracy and resolution of kinect depth data for indoor mapping applications," Sensors, vol. 12, no. 2, pp. 1437-1454, 2012.

[11] N. Franklin, "Language as a means of constructing and conveying cognitive maps," The Construction of Cognitive Maps, pp. 275-295, 1995.

[12] I. Pitt and A. Edwards, "Improving the usability of speechbased interfaces for blind users," in Int. ACM Conf. Assistive Technologies. New York, NY, USA: ACM, 1996, pp. 124-130.

[13] T. Strothotte, S. Fritz, R. Michel, A. Raab, H. Petrie, V. Johnson, L. Reichert, and A. Schalt, "Development of dialogue systems for a mobility aid for blind people: initial design and usability testing," in Proc. 2nd Annu ACM Conf. Assistive Technologies. New York, NY, USA: ACM, 1996, pp. 139144.
[14] A. Fattouh, M. Sahnoun, and G. Bourhis, "Force feedback joystick control of a powered wheelchair: preliminary study," in IEEE Int. Conf. Systems, Man and Cybernetics, vol. 3, Oct. 2004, pp. 2640-2645.

[15] J. Staton and M. Huber, "An assistive navigation paradigm using force feedback," in IEEE Workshop on Advanced Robotics and its Social Impacts (ARSO), Nov. 2009, pp. 119-125.

[16] Novint Technologies Inc, "Novint Falcon," accessed 2013-0618. [Online]. Available: http://www.novint.com/index.php/ novintfalcon

[17] SICK Inc., "LMS100 and LMS111," accessed 2013-06-18. [Online]. Available: http://www.sick.com/us/en-us/home/ products/product_news/laser_measurement_systems/ Pages/lms100.aspx

[18] MSI, "MSI global - notebook and tablet - GT663," accessed 2013-06-18. [Online]. Available: http://www.msi. com/product/nb/GT663.html

[19] H. Fredriksson, "Laser on kinetic operator," Ph.D. dissertation, Luleå University of Technology, Luleå, Sweden, 2010.

[20] K. Hyyppä, "On a laser anglemeter for mobile robot navigation," Ph.D. dissertation, Luleå University of Technology, Luleå, Sweden, 1993.

[21] S. Rönnbäck, "On methods for assistive mobile robots," Ph.D. dissertation, Luleå University of Technology, Luleå, Sweden, 2006.

[22] SenseGraphics AB, "Open source haptics - H3D.org," accessed 2013-06-18. [Online]. Available: http://www.h3dapi.org/

[23] D. C. Ruspini, K. Kolarov, and O. Khatib, "The haptic display of complex graphical environments," in Proc. 24th Annu. Conf. Computer Graphics and Interactive Techniques. New York, NY, USA: ACM Press/Addison-Wesley Publishing Co., 1997, pp. 345-352.

[24] D. Innala Ahlmark, M. Prellwitz, J. Röding, L. Nyberg, and K. Hyyppä, "A haptic obstacle avoidance system for persons with a visual impairment: an initial field trial," to be published.

[25] J. Rantala, K. Myllymaa, R. Raisamo, J. Lylykangas, V. Surakka, P. Shull, and M. Cutkosky, "Presenting spatial tactile messages with a hand-held device," in IEEE World Haptics Conf. (WHC), Jun. 2011, pp. 101-106.

[26] R. Velazquez and S. Gutierrez, "New test structure for tactile display using laterally driven tactors," in Instrumentation and Measurement Technol. Conf. Proc., May 2008, pp. 1381-1386.

[27] A. Yamamoto, S. Nagasawa, H. Yamamoto, and T. Higuchi, "Electrostatic tactile display with thin film slider and its application to tactile telepresentation systems," IEEE Trans. Visualization and Computer Graphics, vol. 12, no. 2, pp. 168177, Mar-Apr 2006.

[28] F. Avanzini and P. Crosato, "Haptic-auditory rendering and perception of contact stiffness," in Haptic and Audio Interaction Design, LNCS, 2006, pp. 24-35. 